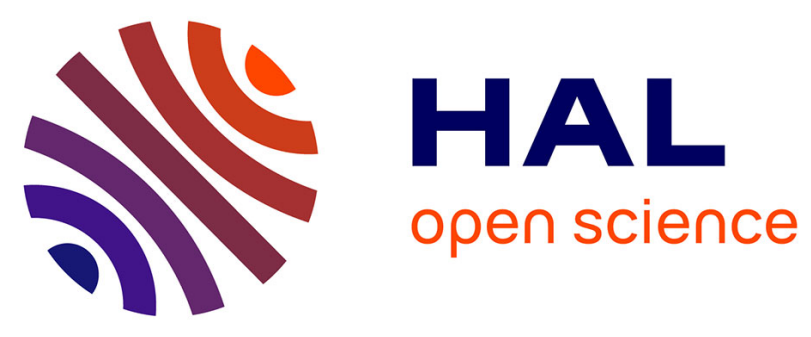

\title{
Robust Design of Pumping Stations in Water Distribution Networks
}

Gratien Bonvin, Sophie Demassey, Welington de Oliveira

\section{To cite this version:}

Gratien Bonvin, Sophie Demassey, Welington de Oliveira. Robust Design of Pumping Stations in Water Distribution Networks. Optimization of Complex Systems: Theory, Models, Algorithms and Applications, 991, pp.957-967, 2019, Advances in Intelligent Systems and Computing, 10.1007/9783-030-21803-4_95. hal-02158548

\section{HAL Id: hal-02158548 \\ https://hal-mines-paristech.archives-ouvertes.fr/hal-02158548}

Submitted on 16 Oct 2019

HAL is a multi-disciplinary open access archive for the deposit and dissemination of scientific research documents, whether they are published or not. The documents may come from teaching and research institutions in France or abroad, or from public or private research centers.
L'archive ouverte pluridisciplinaire HAL, est destinée au dépôt et à la diffusion de documents scientifiques de niveau recherche, publiés ou non, émanant des établissements d'enseignement et de recherche français ou étrangers, des laboratoires publics ou privés. 


\title{
Robust design of pumping stations in water distribution networks
}

\author{
Gratien Bonvin $^{1}$, Sophie Demassey ${ }^{1}$, and Welington de Oliveira ${ }^{1}$ \\ Center for Applied Mathematics, \\ Mines ParisTech, PSL Research University \\ Sophia Antipolis, France \\ \{gratien.bonvin, sophie.demassey, welington.oliveira\}@mines-paristech.fr
}

\begin{abstract}
Restricted to gravity-fed networks, most water network design models minimize investment costs under a static peak water demand scenario. In networks equipped with pumping stations, design models should also account for operation costs incurred by the pump energy consumption that depends on dynamic demand and tariff. Evaluating the lifetime operation costs amounts to solve a large-scale non-convex combinatorial optimization problem for each considered design. In this paper, we address the pressurized water network design problem with a joint optimization of the pump investment and operation costs through a stabilized Benders' decomposition. To reduce the complexity of the operational subproblem, we decompose the scheduling horizon in representative days, and relax the discrete and non-convex components of the hydraulic model. We also evaluate the design robustness on stress-day scenarios and derive feasibility cuts using a dominance argument. Experiments on a typical rural branched water distribution network with one year of historical data show the accuracy of our approximations and the significant savings expected from the optimal pump resizing.
\end{abstract}

Keywords: Pressurized Water Network Design · Stabilized Benders' Decomposition · Mixed Integer Nonlinear Programming.

\section{Introduction}

While the lifetime of pipes and water tanks usually reaches 100 years, the mean lifetime of a pump is closer to 20 years. Operators of water networks must then periodically proceed to the rehabilitation of pumping stations with the characteristics of the other network assets already fixed. The problem is complex because, besides the strategical level - which pump combination to install? - it requires to investigate the operational level - how to operate the installed pumps? - to evaluate and minimize the lifetime costs over the set of pump combinations. Moreover, evaluating the minimum operation costs brings into play, together, dynamic water demand and energy tariff profiles, discrete pump scheduling decisions, non-convex hydraulic laws, and uncertain long-term forecasts. Actually, solving the operational subproblem, known as pump scheduling problem, deterministically on a daily horizon is already considered challenging (see e.g. [7]). 
Optimization methods dedicated to the design of water distribution networks have been proposed for more than four decades. A common approach is to combine an evolutionary algorithm with a hydraulic simulator (see e.g. $[9,10]$ and references therein). The approach deals accurately with the short-term dynamic of the network operation, but it is inherently a heuristic and provides no performance guarantee. Mathematical programming approaches handle the hydraulic explicitly as non-linear constraints to address the pipe layout design problem $[2,4,12]$. They provide guarantee, but neglect the dynamic by evaluating the feasibility of operating the network on a static worst-case water demand scenario.

In this paper, we address the pumping station design problem precisely, with an optimization approach combining the strengths of both previous strategies. While the overall approach is generic, the operational level is presented in the context of the FRD network, a branched network that is described in details in [3]. The approach is built on a decomposable formulation of the problem including the operation scheduling subproblem as a large-scale non-convex mixedinteger non-linear program with uncertain data. We apply a stabilized Benders' decomposition [1] to solve the problem and propose different approximations for the subproblem to reduce its complexity while maintaining some performance guarantees. First, we decompose the multi-year scheduling horizon in a restricted set of seasonal representative days. This common assumption in long-term planning models fits well with the daily periodicity of drinking water distribution, and allows to separate the scheduling subproblem in independent daily subproblems (once a pump configuration is given). As optimizing iteratively each subproblem would remain too time consuming, we propose to relax the integrality constraints and to convexify the hydraulic constraints. This results in convex continuous non-linear programs providing under-estimates of the operation costs and dual information to derive Benders cuts. Second, we handle the long-term uncertainties by enforcing the robustness of the solution on hypothetical stress days characterized, e.g., by a high water demand and the outage of one pump. By disregarding optimality but forcing feasibility on stress days we can, for the class of networks in consideration, aggregate identical pumps and, therefore, reduce the size and complexity of the subproblem. Furthermore, we exhibit a dominance relation between pump combinations that allows to generate multiple feasibility cuts from one infeasible solution. Our experiments on the FRD network show the accuracy of our approximations: the impact of the horizon decomposition is negligible while the continuous convex relaxation induces a deviation of the optimum lower than $4.3 \%$. Finally, the annual savings (in terms of investment and operation costs) expected from the rehabilitation are estimated to $32 \%$.

The paper is organized as follows: Section 2 presents a formulation of the problem. Section 3 describes the stabilized Benders' decomposition and Section 4 the management of infeasibility under the dominance argument. Computational results are provided in Section 5 and some conclusions in Section 6. 


\section{Optimal design of a pumping station}

This section defines our design model, by first describing how the network operates in our application case. This simpler operation model detailed in [3] is a specialization of standard models to the network characteristics [6].

\subsection{Operation of a branched network}

The water distribution network FRD [3] can be represented as a directed graph $\mathcal{G}=(J, L)$. The water flows from a source $r \in J$ to elevated tanks $j \in J_{T} \subseteq J$ through, successively: fixed speed pumps $k \in K \subseteq L$ set in parallel at the pumping station $s \in J$, directed pipes $l \in L_{P} \subseteq L$ connected by junctions $j \in J_{J} \subseteq J$, and pressure reducing valves $l \in L_{V} \subseteq L$. The dynamic state of the system over a given time horizon $\mathcal{T}$ is driven by the water demand $D \in \mathbb{R}_{+}^{J_{T} \times \mathcal{T}}$ at the tanks and is governed by complex hydraulic laws of conservation of flow and pressure through the network. A standard model is defined as

$$
\mathcal{P}_{\mathcal{T}}^{K}=\left\{(x, q, h) \in\{0,1\}^{K \times \mathcal{T}} \times \mathbb{R}_{+}^{L \times \mathcal{T}} \times \mathbb{R}_{+}^{J \times \mathcal{T}} \mid(1)-(9)\right\},
$$

with $x$ the on/off state of the pumps, $q$ the flow through the arcs, and $h$ the head (sum of pressure and elevation in $m$ ) at the nodes, and:

$$
\begin{array}{lr}
\sum_{i j \in L} q_{i j t}=\sum_{j i \in L} q_{j i t}, & t \in \mathcal{T}, j \in J_{J} \\
\sum_{i j \in L} q_{i j t}=S_{j}\left(h_{j t}-h_{j t-1}\right)+D_{j t}, & t \in \mathcal{T}, j \in J_{T} \\
h_{j 0}=h_{j T}=H_{j}^{0}, & j \in J_{T} \\
H_{j}^{\min } \leq h_{j t} \leq H_{j}^{\max }, & t \in \mathcal{T}, j \in J_{T} \\
h_{i t}-h_{j t} \geq 0, & t \in \mathcal{T}, i j \in L_{V} \\
\alpha^{\min } \leq h_{s t}-h_{r t} \leq \alpha^{\max }, & t \in \mathcal{T} \\
Q_{\kappa_{k}}^{\min } x_{k t} \leq q_{k t} \leq Q_{\kappa_{k}}^{\max } x_{k t}, & t \in \mathcal{T}, k \in K \\
h_{i t}-h_{j t} \geq \Phi_{i j}\left(q_{i j t}\right), & t \in \mathcal{T}, i j \in L_{P} \\
h_{s t}-h_{r t} \leq \Psi_{\kappa_{k} t}\left(q_{k t}\right)+M\left(1-x_{k t}\right), & t \in \mathcal{T}, k \in K .
\end{array}
$$

In this model, the time horizon is discretized $\mathcal{T}=\{1, \ldots, T\}$ with a resolution of typically $1 \mathrm{hr}$ or $2 \mathrm{hrs}$ in which the system is assumed to operate in steady state. Flows $q_{l t}$ and demands $D_{j t}$ are given in volume (in $m^{3}$ ) for the duration of a time step $t \in \mathcal{T}$. Constraints (1) and (2) enforce the conservation of flow at junctions and tanks. In (2), a tank $j \in J_{T}$ is assumed to be a vertical cylinder of area $S_{j}\left(\right.$ in $m^{2}$ ). Constraints (3) fix the initial and final volumes and Constraints (4) the security limits with $0 \leq H_{j}^{\min } \leq H_{j}^{0} \leq H_{j}^{\max }$ (in $m^{3}$ ). Constraints (5) model the pressure reduction at the valves. Constraints (6) enforce bounds $0<$ $\alpha^{\min } \leq \alpha^{\max }$ on the head increase to limit leaks for instance. Let $\kappa_{k}$ denote the class of pump $k \in K$. Constraints (7) limit the pump operation range, given $0<Q_{\kappa}^{\min } \leq Q_{\kappa}^{\max }$ (in $m^{3}$ ), and bind flow values and pump activation states. 
Constraints (8) models the head loss due to friction in pipes. For each pipe $i j \in$ $L_{p}$, the head loss-flow coupling function $\Phi_{i j}$ can be accurately approximated by a quadratic function $\Phi_{i j}(q)=A_{i j} q+B_{i j} q^{2}$ with $A_{i j} \geq 0, B_{i j} \geq 0$. Constraints (9) synchronize (given a large enough $M$ value) the head increase of each active pumps. Function $\Psi_{\kappa t}$ depends on the manufacture characteristics $\kappa$ and on the ageing $t$ of the pump. It can be accurately fitted from operating points as a quadratic function $\Psi_{\kappa t}(q)=\alpha_{\kappa t}-\beta_{\kappa t} q^{2}$, with $\alpha_{\kappa t} \geq 0$ and $\beta_{\kappa t} \geq 0$. We highlight that the head-flow coupling constraints (8) and (9) are actually equalities in the original - thus non-convex - formulation of the pump scheduling problem, but it is shown in [3] that the optimality gap of this relaxation is small.

Finally, the financial cost of operation plan $(x, q, h)$ is mainly incurred by the purchase of the electricity consumed by pumping, namely:

$$
C_{\mathcal{T}}^{K}(x, q, h)=\sum_{t \in \mathcal{T}} C_{t} \sum_{k \in K} \Gamma_{\kappa_{k} t}\left(x_{k t}, q_{k t}\right),
$$

with $C_{t} \geq 0$ the actualized electricity price on period $t$ and $\Gamma_{\kappa t}$ the power consumption function for each active pump of class $\kappa$ and ageing $t$ defined as a linear fit $\Gamma_{\kappa t}\left(x_{k t}, q_{k t}\right)=\lambda_{\kappa t} x_{k t}+\mu_{\kappa t} q_{k t}$.

\subsection{Robust design and relaxed operation}

In the considered water network design problem, only the set $K$ of pumps must be sized in a way to satisfy the future water demand $D$ and to minimize the global cost - sum of the actualized investment and operation costs - over a life span $\mathcal{T}$ of typically 20 years. The number of pumps in $K$ is limited by the capacity $N \in \mathbb{N}_{*}$ of the pumping station. Each pump is selected from a given set of candidate classes $\kappa \in \mathcal{K}$ and acquired new at time $t=0$ at a fixed investment cost $I_{\kappa} \geq 0$. We assume that the maximal efficiency and the head increase at constant flow for all pumps decrease from $1 \%$ each year, according to the empirical ageing model of [5]. Under this hypothesis, we assume in the definition of $\Phi_{i j}$ that $\alpha_{\kappa t}=\left(1-\frac{v_{t}}{100}\right) \alpha_{\kappa 0}$ and $\beta_{\kappa t}=\left(1-\frac{v_{t}}{100}\right) \beta_{\kappa 0}$ for any time $t \in \mathcal{T}$ with $v_{t}$ the duration from time 0 to $t$ in years. Also, because the power consumption can alternatively be formulated as the product of the flow and the head increase divided by the efficiency, we assume that it does not change in time, i.e. $\lambda_{\kappa t}=\lambda_{\kappa}$ and $\mu_{\kappa t}=\mu_{\kappa}$ for $t \in \mathcal{T}$ in the definition of $\Gamma_{\kappa t}$.

Assuming that the water demand $\left(D_{t}\right)_{t \in \mathcal{T}}$ and the electricity price $\left(C_{t}\right)_{t \in \mathcal{T}}$ are given, the optimal design problem is to find a set $K$ of at most $N$ pumps of classes in $\mathcal{K}$ and an operation plan $(x, q, h) \in \mathcal{P}_{\mathcal{T}}^{K}$ of minimum investment and operation costs. As the life span extends into years while the operation model requires a time resolution in minutes or hours, this model is not practical due to its complexity, its dimension and the stochasticity of the water demand. To address these issues, we formulate a robust variant of the problem where the time horizon is decomposed into a given set of regular (resp. peak) days $d \in \mathcal{D}_{\mathcal{R}}$ (resp. $d \in \mathcal{D}_{\mathcal{P}}$ ). Each day $d \in \mathcal{D}=\mathcal{D}_{\mathcal{R}} \cup \mathcal{D}_{\mathcal{P}}$ is characterized by water demand and electricity tariff profiles over the daily time horizon $\mathcal{T}^{d}$ which 
are representative for a number $L_{d}$ of days over the life span $\mathcal{T}$. Thanks to Constraints (3) (which forces the tank's water level at the end of the day to be equal to that of the beginning of the day) the subproblem of determining the minimum cost operation plan $(x, q, h) \in \mathcal{P}_{\mathcal{T}}^{K}$ associated to a pump combination $K$ is decomposed into independent daily subproblems:

$$
\min _{(x, q, h) \in \mathcal{P}_{\mathcal{T}}^{K}} C_{\mathcal{T}}^{K}(x, q, h)=\sum_{d \in \mathcal{D}} L_{d} \min _{\left(x^{d}, q^{d}, h^{d}\right) \in \mathcal{P}_{\mathcal{T}^{d}}^{K}} C_{\mathcal{T}^{d}}^{K}(x, q, h) .
$$

Because optimizing on $\mathcal{P}_{\mathcal{T}^{d}}^{K}$ remains challenging, we propose to relax some operational constraints which are not structural for the long term horizon. We consider a different relaxation $\mathcal{R}_{\mathcal{T}^{d}}^{K}$ whether $d$ is a regular or peak day. The purpose of peak days is to enforce the robustness of the solutions by simulating stress operation conditions, including a high demand. We check the feasibility of the pump combinations under these extreme conditions but, if feasible, we neglect the associated operation costs as these conditions are considered as exceptional. More specifically, we consider the sum in (11) only over the index set of regular days $\mathcal{D}_{\mathcal{R}}$, and we use the peak days to check feasibility (of the pump configuration), as detailed in Section 3 below. In such stress days we also ignore the minimum flow constraints in (7) by setting $Q_{\kappa}^{\min }=0$. Under these assumptions, all the pumps in a class can be assumed to operate equally and the size of model $\mathcal{R}_{\mathcal{T}^{d}}^{K}$ can be reduced accordingly, by aggregating the variables per pump class and by relaxing the integrality constraints on the aggregated state variables for each class $\kappa \in \mathcal{K}$ such that $\alpha_{\kappa 0} \geq \alpha^{\max }$.

To allow a quick computation of the operation costs of a pump combination over the regular days, as well as the use of dual information in the overall solution algorithm described in the next section, we define $\mathcal{R}_{\mathcal{T}^{d}}^{K}$ as the continuous relaxation of $\mathcal{P}_{\mathcal{T}^{d}}^{K}$ for any regular day $d \in \mathcal{D}_{R}$. I.e., the feasible sets $\mathcal{P}_{\mathcal{T}^{d}}^{K}$ in (11) are replaced with $\mathcal{R}_{\mathcal{T}^{d}}^{K}$.

\subsection{Pump investment variables, constraints and costs}

As the considered network has only one pumping station, a pump combination is uniquely determined by the number of pumps of each class it contains. An alternative representation, with more symmetries but that is better suited to Benders cut generation, is given by $y_{\kappa n} \in\{0,1\}$ for $\kappa \in \mathcal{K}$ and $n \in\{1, \ldots, N\}$ such that $y_{\kappa n}=1$ if and only if the combination has at least $n$ pumps of class $\kappa$. For a fixed ordering of the pumps, each combination corresponds to exactly one binary vector $y$ such that

$$
\sum_{\kappa \in \mathcal{K}} \sum_{n=1}^{N} y_{\kappa n} \leq N, \quad y_{\kappa n} \leq y_{\kappa n-1}, \quad \text { for all } \kappa \in \mathcal{K} \text { and } n=2 \ldots, N .
$$

Let $N_{\kappa}(y)=\sum_{n=1}^{N} y_{\kappa n}$ be the number of pumps of class $\kappa$ in the combination $y$. The investment cost of acquiring $N_{\kappa}(y)$ pumps of class $\kappa$ is $I_{\kappa} N_{\kappa}(y)$, with given $I_{\kappa} \geq 0$. Having defined all the elements related to the variables of investment 
and operation, we are now in position to state the considered formulation for the robust design optimization problem:

$$
\left\{\begin{array}{lll}
\min _{y, x, q, h} \sum_{\kappa \in \mathcal{K}} I_{\kappa} N_{\kappa}(y)+\sum_{d \in \mathcal{D}_{\mathcal{R}}} L_{d} C_{\mathcal{T}_{d}}^{\mathcal{K}}\left(x^{d}, q^{d}, h^{d}\right) & \\
\text { s.t. } & y \text { binary satisfying }(12) \text { and } x^{d} \leq y & \forall d \in \mathcal{D}_{\mathcal{R}} \cup \mathcal{D}_{\mathcal{P}} \\
& \left(x^{d}, q^{d}, h^{d}\right) \in \mathcal{R}_{\mathcal{T}_{d}}^{\mathcal{K}} & \forall d \in \mathcal{D}_{\mathcal{R}} \cup \mathcal{D}_{\mathcal{P}} .
\end{array}\right.
$$

The constraint $x^{d} \leq y$ means that a pump is operational at day $d$ if the configuration $y$ accounts its installation.

\section{Benders' decomposition}

Problem (13) has a decomposable structure: we can separate the investment variables $y$ from the operational ones $(x, q, h)$ to rewrite $(13)$ as

$$
\min _{y} f(y) \quad \text { s.t. } \quad y \text { binary satisfying }(12) \text { and } c(y)=0 .
$$

In this formulation, the objective function $f$ is

$$
f(y)=\sum_{\kappa \in \mathcal{K}} I_{\kappa} N_{\kappa}(y)+\sum_{d \in \mathcal{D}_{\mathcal{R}}} L_{d}\left\{\begin{array}{l}
\min _{x^{d}, q^{d}, h^{d}} C_{\mathcal{T}^{d}}^{K}\left(x^{d}, q^{d}, h^{d}\right) \\
\text { s.t. } \quad\left(x^{d}, q^{d}, h^{d}\right) \in \mathcal{R}_{\mathcal{T}_{d}}^{\mathcal{K}} \\
x^{d} \leq y \quad \forall d \in \mathcal{D}_{\mathcal{R}} .
\end{array} \quad \forall d \in \mathcal{D}_{\mathcal{R}}\right.
$$

Peak days are handled by the constraint function $c$ :

$$
c(y)=\left\{\begin{array}{l}
0 \text { if there exist }\left(x^{d}, q^{d}, h^{d}\right) \in \mathcal{R}_{\mathcal{T}_{d}}^{\mathcal{K}} \text { s.t. } x^{d} \leq y \forall d \in \mathcal{D}_{\mathcal{R}} \cup \mathcal{D}_{\mathcal{P}} \\
\infty \text { otherwise. }
\end{array}\right.
$$

In the process of solving (14), both $f$ and $c$ are approximated by cuts.

Benders cuts. Note that function $f$ is convex but nonsmooth. Therefore, optimality cuts for (14) is nothing but linearization of $f$, computed by making use of subgradients. Let $\tilde{y}$ be a given combination of pumps. Then a subgradient $\tilde{s}$ of $f$ at $\tilde{y}$ is a vector of same dimension as $\tilde{y}$ constructed from the dual variables associated to the constraints $x^{d} \leq y$, for all $d \in \mathcal{D}_{\mathcal{R}}$, as described in [1, Lemma 1]. The optimality cut is thus given by $f(\tilde{y})+\langle\tilde{s}, \cdot-\tilde{y}\rangle$ and satisfies (due to convexity of $f) f(\tilde{y})+\langle\tilde{s}, y-\tilde{y}\rangle \leq f(y)$ for all $y$.

Feasibility cuts. Suppose that $\tilde{y}$ is infeasible for problem (14). In order to exclude such a point from the set of candidate solutions for (14) we consider the following linear constraint $\sum_{\kappa \in \mathcal{K}} y_{\kappa N_{\kappa}(\tilde{y})+1} \geq 1$, with convention $y_{\kappa N+1}=0$.

\subsection{Stabilized cutting-plane algorithm}

Let $\ell$ be an iteration counter and $\underline{z}^{\ell}$ (resp. $\bar{z}^{\ell}$ ) be the lower (resp. upper) bound available at iteration $\ell$ for the optimal value of (14). We initialize $\underline{z}^{0}=0$ and $\bar{z}^{0}=\infty$ in the stabilized Benders' decomposition of [1] to generate a sequence $\left\{y^{\ell}\right\}$ of be the trial points for (14). We split such a sequence according to feasibility of its elements: $\mathcal{O}^{\ell}=\left\{\iota \in\{0, \ldots, \ell\}: c\left(y^{\iota}\right)=0\right\}$ and $\mathcal{F}^{\ell}=\{0, \ldots, \ell\} \backslash \mathcal{O}^{\ell}$. 
Note that $\mathcal{F}^{\ell}$ gathers (up to iteration $\ell$ ) the points that have been proved infeasible for problem (14). By setting $\ell=0$, starting with a vector $y^{0}$ and defining the incumbent point $\hat{y}^{0}=y^{0}$, our variant of [1, Alg. 4] defines trial points by iteratively solving the following MILP:

$$
y^{\ell+1} \in\left\{\begin{array}{lll}
\arg \min _{y}\left\langle\frac{1}{2} \mathbf{1}-\hat{y}^{\ell}, y\right\rangle & \\
\text { s.t. } & y \text { binary satisfying }(12) & \\
& f\left(y^{\iota}\right)+\left\langle s^{\iota}, y-y^{\iota}\right\rangle \leq\left(\underline{z}^{\ell}+\bar{z}^{\ell}\right) / 2 & \forall \iota \in \mathcal{O}^{\ell} \\
& \sum_{\kappa \in \mathcal{K}} y_{\kappa N_{\kappa}\left(y^{\iota}\right)+1} \geq 1 & \forall \iota \in \mathcal{F}^{\ell} .
\end{array}\right.
$$

If this master program is infeasible, then $\underline{z}^{\ell+1}=\left(\underline{z}^{\ell}+\bar{z}^{\ell}\right) / 2$ is a valid lower bound for (14). Otherwise, we set $\underline{z}^{\ell+1}=\underline{z}^{\bar{\ell}}$ and check feasibility of $z^{\ell+1}$ : if it is infeasible we set $\mathcal{F}^{\ell+1}=\mathcal{F}^{\ell} \cup\{\ell+1\}$ (a new feasibility cut is added). On the other hand, if $c\left(z^{\ell+1}\right)=0$ we then update $\mathcal{O}^{\ell+1}=\mathcal{O}^{\ell} \cup\{\ell+1\}$ (a new Benders cut is added), compute $f\left(z^{\ell+1}\right)$ and a subgradient $s^{\ell+1}$, and set $\bar{z}^{\ell+1}=$ $\min \left\{\bar{z}^{\ell}, f\left(y^{\ell+1}\right)\right\}$. If $\bar{z}^{\ell}>f\left(y^{\ell+1}\right)$ then the algorithm updates the incumbent point: $\hat{y}^{\ell+1}=y^{\ell}$, otherwise it remains unchanged. In any case, the algorithm updates $\ell=\ell+1$ and repeats this process until $\left(\bar{z}^{\ell}-\underline{z}^{\ell}\right) / \bar{z}^{\ell} \leq$ tol, for a given tolerance tol $>0$. We refer to [1] for further details on the algorithm.

\section{Infeasibility, dominance and fault tolerance}

In this section, we show how to accelerate the convergence of the solution algorithm by using a dominance relation on the set of combinations to generate more than one feasibility cut at a time, including in a preprocessing step. To simplify the presentation, we fairly assume that a combination which is infeasible for a regular day is also infeasible for a peak day. We finally integrate to the definition of peak days a model of robustness to pump failures.

\subsection{Dominance}

By definition, the maximal flow a pump combination $y$ can deliver for a given head increase $\alpha=h_{s}-h_{r}$ corresponds to activate all $N_{\kappa}(y)$ pumps of each class $\kappa \in \mathcal{K}$ with $\alpha_{\kappa 0}>\alpha$. It is thus equal to $Q_{y}(\alpha)=\sum_{\kappa \in \mathcal{K}} N_{\kappa}(y) \sqrt{\max \left(0, \frac{\alpha_{\kappa 0}-\alpha}{\beta_{\kappa}}\right)}$. We say that a combination $y$ dominates $y^{\prime}$, and we note $y^{\prime} \preceq y$, if $Q_{y^{\prime}} \leq Q_{y}$ on the allowed operation range $\left[\alpha^{\min }, \alpha^{\max }\right]$. The following proposition shows that all combinations dominated by an infeasible combination are infeasible too.

Proposition 1. For any combinations $y$ and $y^{\prime}$ and peak day $d \in \mathcal{D}_{\mathcal{P}}$, if $y^{\prime} \preceq y$ and $y$ is infeasible for (14), so is $y^{\prime}$.

Proof. For any combination $y$, function $Q_{y}$ is strictly decreasing with the head increase, then $A_{y}=\left\{(q, \alpha) \in \mathbb{R}^{2} \mid 0 \leq q \leq Q_{y}\left(\alpha^{\min }\right), \alpha^{\min } \leq \alpha \leq Q_{y}^{-1}(q)\right\}$ identifies the set of pairs of total flow and head increase which can be operated by $y$ at any time $t$ (because we relaxed lower bound $Q_{\kappa}^{\min }$ on peak days). Then for any point in $\mathcal{R}_{d}\left(y^{\prime}\right)=\left\{\left(x^{\prime}, q^{\prime}, h^{\prime}\right) \in \mathcal{R}_{\mathcal{T}_{d}}^{\mathcal{K}}\right.$ s.t. $\left.x^{d} \leq y^{\prime} \forall d \in \mathcal{D}_{\mathcal{P}}\right\}$, which is built on a sequence of $\mathcal{T}_{d}$ elements of $A_{y^{\prime}} \subseteq A_{y}$, there exists another point $\mathcal{R}_{d}(y)$. 


\subsection{Generation of feasibility cuts}

Suppose that, in the cutting-plane algorithm, a candidate combination $y^{\ell+1}$ is proved to be infeasible on some peak day. Proposition 1 implies that several feasibility cuts can be generated at the same iteration instead of a single one: it amounts to search for combinations that are dominated by $y^{\ell+1}$. Dominance clearly defines a partial order relation on the set of combinations. The dominance of $y^{\prime}$ by $y$ can be checked by proving that $Q_{y}-Q_{y^{\prime}} \geq 0$ at each point of nondifferentiability $\left\{\alpha^{\min }, \alpha^{\max }\right\} \cup\left\{\alpha_{\kappa} \mid \kappa \in \mathcal{K}\right\}$. However, the class of dominated combinations can reasonably be computed only for small values of $|\mathcal{K}|$ or $N$. We propose to compute it in a heuristic way by observing that, if $p_{\kappa}$ denotes the power of a pump $\kappa$ at its maximal efficiency then the reference power $P(y)=$ $\sum_{\kappa \in \mathcal{K}} N_{\kappa}(y) p_{\kappa}$ of $y$ is likely greater than the reference power of the combinations it dominates. In our implementation, we first compute and sort the list $\mathcal{C}$ of combinations of at most $N$ pumps by increasing order of reference power. When $y$ is proved to be infeasible, we iterate on the combinations with lower reference power, by decreasing order, and check if they are dominated. Each dominated combination is then used to enlarge the set $\mathcal{F}^{\ell}$ in (16) indexing feasibility cuts.

As a preprocessing step for the algorithm, to accelerate its convergence, we also propose to initialize $\mathcal{F}^{0}$ by greedily evaluating the list $\mathcal{C}$. We iteratively pick up a combination $y \in \mathcal{C}$ of median value $P(y)$ and check the feasibility of the continuous relaxation of $\mathcal{R}_{\mathcal{T} d}^{K}$ on peak days $d \in \mathcal{D}_{\mathcal{P}}$. If $y$ is infeasible, then we updated $\mathcal{F}^{0}$ as previously. Otherwise, we remove all $y^{\prime}$ from $\mathcal{C}$ with $P\left(y^{\prime}\right) \geq P(y)$ s.t. $y \preceq y^{\prime}$ as they are likely feasible too.

\subsection{Robustness to a pump outage}

Finally, we propose to add another degree of robustness to the design problem by simulating the inoperability of one pump on peak days (due to possible failure or maintenance). To that purpose, we redefine that a combination $y$ is feasible for (14) if all variations of $y$ defined by setting off one pump (of any type) on peak days are feasible. This new assumption only impacts (and speeds up) the algorithms described above to initialize and update the index set $\mathcal{F}^{\ell}$ of feasibility cuts.

\section{Numerical assessments on a real-life instance}

In this section, we consider the rural drinking water distribution network $F R D$ investigated in [3]. The resulting design problem (13) were constructed with data from the historical demand profiles of the year 2013 (see [3] for more details). We assume that all the 6 existing pumps should be replaced by at most $N=6$ new ones selected in the KSB manufacturer catalog [8]. We have preselected a set $\mathcal{K}$ of 19 classes of pumps compatible with the network allowed range of pressure $\left[\alpha_{\min }=91, \alpha_{\max }=140\right]$. For each class $\kappa \in \mathcal{K}$, we interpolated curves $\Psi_{\kappa}$ and $\Gamma_{\kappa}$ from the catalog data and estimated the investment cost $I_{\kappa}=4400\left(\frac{p_{\kappa}}{74.6}\right)^{0.67}+$ 
$19300\left(\frac{p_{\kappa}}{52}\right)^{0.77}$ following ([11], Table 9-50). Considering a planning horizon of $T=20$ years, we built a set $\mathcal{D}_{\mathcal{R}}$ of 12 regular days, each representative of a week day or a week-end in six 2-months periods (January-February, March-April,...) of any year. For each regular day $d \in \mathcal{D}_{\mathcal{R}}$, we define $L_{d}=L_{d}^{0} \sum_{l=1}^{20}(1+\tau)^{1-l}$ with $L_{d}^{0}$ the number of days represented by $d$ in 2013 and $\tau=5 \%$ the discount rate. Demand and tariff profiles $D^{d}$ and $C^{d}$ for a regular day $d$ are computed in average over the $L_{d}^{0}$ represented days of 2013 . The unique peak day is built from the day in 2013 with the highest instantaneous demand and by initializing the tanks at their minimum level $H^{\text {min }}$. We fix the time resolution (duration of the time steps) to 2 hours for regular days and to 4 hours for peak days.

The computations were performed on a Xeon E5-2650V4 2.2GHz with 254 GB RAM. The algorithms were implemented in Python (with tol $=10^{-3}$ ), and the master and slave programs solved with Gurobi 7.0.2.

\subsection{Numerical results}

The preprocessing algorithm identified 1,902 infeasible combinations of at most 5 pumps (among 42,504) in 275 seconds, $83 \%$ of the time being to compare combinations. This resulted in an initial set $\mathcal{F}^{0}$ of 20,757 infeasible combinations of at most 6 pumps, which represent $96 \%$ of $\mathcal{F}^{\ell}$ at the last iteration $\ell$ of the stabilized cutting-plane algorithm. This suggests that the preprocessing algorithm separates feasible and infeasible combinations with good accuracy.

The cutting-plane algorithm ran in 732 seconds and 37 iterations (28 infeasible candidates). In average, the time of one iteration can be roughly decomposed in 12 seconds for solving the master program, 6 seconds for the twelve regular slave programs (which could be reduced by parallelization), and 3 seconds for the peak slave program and to compute the dominated infeasible combinations.

To evaluate the quality of our approximations, we computed the non-relaxed operation cost of the optimal combination $y^{*}$ when optimizing on the original operation scheduling model $\mathcal{P}_{\mathcal{T}}^{K}$ (using the heuristic in [3]) on the 12 regular days of 2013 and on the 365 days of year 2013. By applying the discount rate, we get the operation costs over the 20 years: 460,388 euros in the former case and 460,247 in the latter case. This negligible deviation suggests that the chosen number of representative days is enough. By adding the investment costs on the 12-day case, we get a lifetime cost of 598,748 euros. Compared to the final lower bound $\underline{z}=570,898$, it gives an optimality gap of $4.3 \%$ which confirms that the continuous relaxation $\mathcal{R}_{d}$ offers a good estimate of the operation cost.

Finally, we compared the operation of network FRD before and after resizing the pumping station according to the optimal combination $y^{*}$. First, the number of pumps is reduced from 6 to 5 pumps and the average reference power from 127 to $66 \mathrm{~kW}$. The purchase cost of the new combination is then half the present value of the installed combination. Second, the operation costs for year 2013, when estimated with $\mathcal{P}_{\mathcal{T}}^{K}$, are reduced by $24.3 \%$, a reduction that is mainly driven by a better usage of the pumps $(+18.9 \%$ of the average efficiency $)$ which proves the better adequation to the demand. 


\section{Conclusion}

In this paper, we tackled the water network design problem in the context of networks equipped with pumping stations, where the operation costs must be estimated dynamically in addition to the investment costs. We formulated different approximations to estimate the operation costs to be embedded in a stabilized Benders' decomposition approach. We also ensured a certain robustness of the solutions to stress operation conditions and derived dominance arguments to accelerate the cutting-plane algorithm. Experimented on a realistic instance, the approximations turned to be accurate and the algorithm fast. While some features of our implementation are specific to the FRD network, and more generally to a class of branched network defined in [3], the method can be generalized to a variety of water networks after identifying an accurate continuous relaxation for the operation problem. The potential higher complexity and larger optimality gap should however be evaluated in practice.

\section{References}

1. van Ackooij, W., Frangioni, A., de Oliveira, W.: Inexact stabilized benders' decomposition approaches with application to chance-constrained problems with finite support. Computational Optimization and Applications 65(3), 637-669 (2016)

2. Alperovits, E., Shamir, U.: Design of optimal water distribution systems. Water Resources Research 13(6), 885-900 (1977)

3. Bonvin, G., Demassey, S., Le Pape, C., Mazi, N., Mazauric, V., Samperio, A.: A convex mathematical program for pump scheduling in a class of branched water networks. Applied Energy 185, 1702 - 1711 (2017)

4. Bragalli, C., D'Ambrosio, C., Lee, J., Lodi, A., Toth, P.: On the optimal design of water distribution networks: a practical MINLP approach. Optimization and Engineering 13(2), 219-246 (2012)

5. Bunn, S.: Ageing pump efficiency: The hidden cost thief? In: Distribution Systems Symposium \& Exposition (2009)

6. Burgschweiger, J., Gnädig, B., Steinbach, M.: Optimization models for operative planning in drinking water networks. Opt. and Eng. 10(1), 43-73 (2009)

7. D'Ambrosio, C., Lodi, A., Wiese, S., Bragalli, C.: Mathematical programming techniques in water network optimization. European J. of Operational Research 243(3), $774-788(2015)$

8. KSB: Multitec : High-pressure pumps in ring-section design - booklet with performance curves., https://shop.ksb.com/

9. Marques, J., Cunha, M., Savi, D.A.: Multi-objective optimization of water distribution systems based on a real options approach. Environmental Modelling \& Software 63, 1 - 13 (2015)

10. Murphy, L., Dandy, G., Simpson, A.: Optimum design and operation of pumped water distribution systems. In: Conf. on Hydraulics in Civil Engineering (1994)

11. Perry, R., Green, D., Maloney, J.: Perrys chemical engineers handbook 7th ed. McGraw-Hill (1997)

12. Raghunathan, A.U.: Global optimization of of nonlinear network design. SIAM Journal on Optimization 23(1), 268-295 (2013) 